\title{
Low Frequency Spherical Vector Hydrophone Based on the Geophone
}

\author{
Shuang Liü, ${ }^{1, a}$, Yu Lan ${ }^{2, b}$ and Qi Li ${ }^{3}$ \\ ${ }^{1,2,3}$ Acoustic Science and Technology Laboratory, Harbin Engineering University, Harbin, China, \\ 150001 \\ ${ }^{1,2,3}$ College of Underwater Acoustic Engineering, Harbin Engineering University, Harbin, China, \\ 150001 \\ a163liushuang@163.com, ' lanyu@hrbeu.edu.cn
}

Keywords: Low frequency; vector hydrophone; sleeved rubber spring; geophone

\begin{abstract}
In this paper, a new type of low frequency spherical vector hydrophone is designed. It can be directly installed on the underwater platform and sense the underwater acoustic particle velocity. The sensing element is a moving-coil geophone. The paper described the structure of the spherical vector hydrophone, analyzed the modal of spherical shell and calculated the influence of the size and material parameters on the stiffness of spherical sleeved rubber spring. In the test frequency range, the sensitivity of vector hydrophone is about - 205dB. The ration of the maximum and the minimum in the directivity pattern is greater than $20 \mathrm{~dB}$.
\end{abstract}

\section{Introduction}

With the development of underwater acoustic technology, researchers pay more attention to the low frequency underwater acoustic detection. Because the directivity of the vector hydrophone has no relation with the frequency and is cosine pattern, using the vector hydrophone to receive acoustic wave can reduce the size of underwater wet end in low frequency. Besides the directivity of the vector hydrophone, the sensitivity of the vector hydrophone is another important technical parameter. The sensitivity of hydrophone depends on the internal sensors and the acoustic quantity that the vector hydrophone measures. At present, the vector hydrophone usually senses the particle acceleration and particle velocity signals under the water[1]. They are all underwater inertial sensors. This kind of vector hydrophone is first built by Leslie[2]. The response of acoustic vector hydrophone based on acceleration sensor increases with increasing frequency at a rate of $6 \mathrm{~dB} /$ oct. But the sensitivity curve of acoustic vector hydrophone based on moving-coil geophone is a horizontal line. So the latter is more suitable for measuring low frequency signals.

A low frequency spherical vector hydrophone based on the moving-coil geophone is designed in this paper. A spherical vector hydrophone is placed in a rigid spherical shell and the acoustically transparent elastic rubber is filled in the space between the spherical shell and hydrophone to act as the suspension component of acoustic vector hydrophone.

\section{Structure of Spherical Vector Hydrophone}

The structure diagram of vector hydrophone can be known in Fig.1.

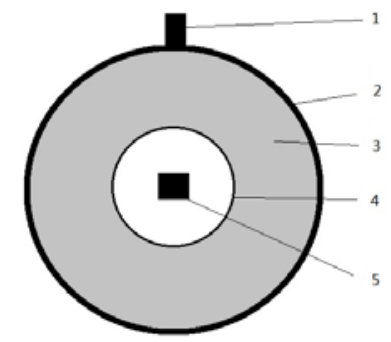

Fig.1 Structural diagram of acoustic vector hydrophone 
In Fig.1, 1 is installing rod of acoustic vector hydrophone, 2 is the rigid spherical shell, 3 is the acoustically transparent elastic rubber, 4 is the spherical vector hydrophone and 5 is the moving-coil geophone inside the vector hydrophone.

The vibration system diagram Fig.2 can be obtained easily from Fig.1.

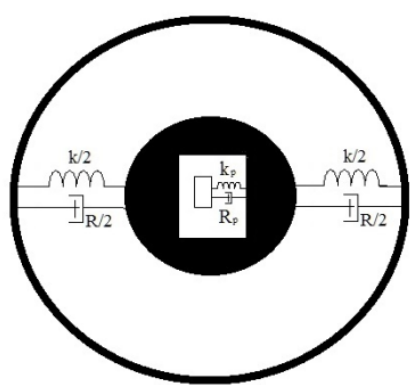

Fig.2 Vibration system diagram of acoustic vector hydrophone based on the geophone

The dynamics of the system were described by McConnell, when an inertial sensor such as a moving-coil geophone was imbedded in the sphere[3]

$$
\begin{aligned}
& \frac{V_{s}}{V_{o}}=-\frac{m_{o}+m_{i}}{m_{c}+m_{i}}\left[\left(1-2 j \xi_{o} \frac{\omega_{o}}{\omega}-\frac{\omega_{o}^{2}}{\omega^{2}}\right) \times\left(1-2 j \xi_{t} \frac{\omega_{t}}{\omega}-\frac{\omega_{t}^{2}}{\omega^{2}}\right)-\frac{m_{t}}{m_{c}+m_{i}} \times\left(2 j \xi_{t} \frac{\omega_{t}}{\omega}+\frac{\omega_{t}^{2}}{\omega^{2}}\right)\right]^{-1} \\
& m_{i}=\frac{2}{3} \pi a^{3} \rho_{c} \frac{1+2\left(a / a_{c}\right)^{3}}{1-\left(a / a_{c}\right)^{3}}
\end{aligned}
$$

$V_{s}$ and $V_{o}$ are the velocity of vector hydrophone and free-field particle velocity under the water respectively. The $R$ and $k$ are the damping and stiffness of acoustically transparent rubber. $R_{p}$ and $k_{p}$ are the damping and stiffness of the coil of the geophone. And $m_{t}$ is the mechanical mass of geophone. So the resonance frequency and damping factor of geophone are $\omega_{t}$ and $\xi_{t}$. They are defined as $\omega_{t}$ $=\left(k_{p} / m_{t}\right)^{1 / 2}$ and $\xi_{t}=R_{p} / 2 m_{t} \omega_{t} . m_{i}$ is the induce mass, $a$ and $a_{c}$ are the inner radius and outer radius of rubber. From Eq.1 and Eq.2, the dynamic characteristics of vector hydrophone can be known.

\section{The Modal Analysis of Rigid Spherical Shell}

Because the vector hydrophone is a kind of inertial sensor, the vibration of the shell will affect the acoustic signal received. To avoid the negative influence of spherical shell modal on the internal vector hydrophone, the modal analysis of the spherical shell was calculated by ANSYS software in order to make the modal frequency outside the operating frequency bandwidth of acoustic vector hydrophone. To make the acoustic vector hydrophone high operating reliability, the material of rigid spherical shell is steel, and the acoustically transparent elastic rubber is filled in the space between the spherical shell and the spherical vector hydrophone.

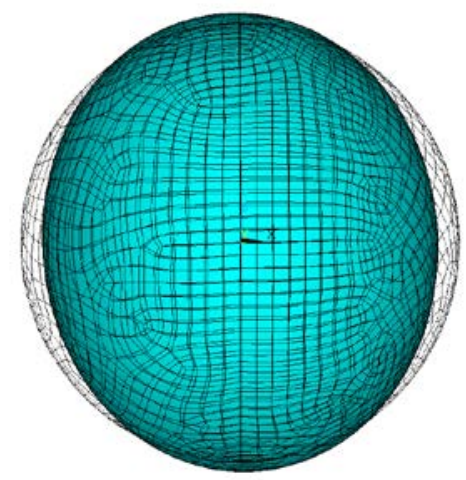

Fig.3 The deformation figure of steel spherical shell at $4112 \mathrm{~Hz}$ 
A three dimensional steel spherical shell model is established. Its radius is $150 \mathrm{~mm}$, and the shell thickness is $5 \mathrm{~mm}$.

Figure 3 is the deformation figure at $4112 \mathrm{~Hz}$ by calculating the modal of steel spherical shell. And it is the lowest modal frequency. As long as the work frequency is far less than the lowest modal frequency, that would not have big influence on the sensitivity of vector hydrophone.

\section{The Stiffness of Spherical Acoustically Transparent Elastic Rubber}

Because the acoustically transparent rubber is regarded as the spring that suspends the acoustic vector hydrophone. So it determines the lower limit operating frequency of acoustic vector hydrophone.

It can be known that when the acoustic vector hydrophone is vibrating in the spherical shell, the inner surface of spherical rubber moves with acoustic vector hydrophone together, and the outside surface of spherical rubber is in the stationary state as a result of the limitation of steel shell from Fig.1. So the spherical rubber can be seen the sleeved rubber spring. The finite element method can be used to calculate the stiffness.

Usually if the shore hardness is known, the shear modulus of rubber can be obtained[4]

$$
G \approx 0.117 e^{0.034 H S}
$$

$H S$ is the shore hardness. The unit of $G$ is MPa. From the above content, if the dimensions of spherical rubber are determined, the stiffness can be worked out by ANSYS software from measuring the shore hardness of rubber.

A three dimensional finite element model of spherical sleeved rubber spring is constructed. The inner radius is $70 \mathrm{~mm}$, and the outer radius is $145 \mathrm{~mm}$. The analysis applies to using the Mooney Rivlin option with the element solid 186 and $G=272000 \mathrm{~Pa}$.

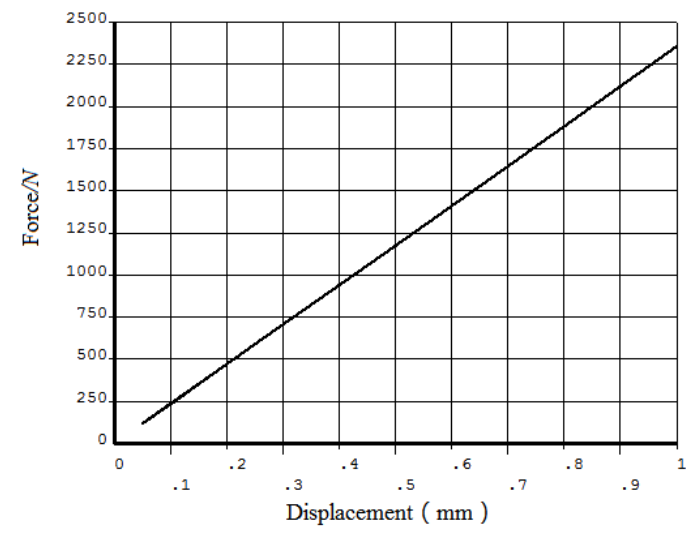

Fig.4 Curve of stiffness

From Fig.4 it can be known that the stiffness is a constant when the displacement is small. But in fact, the stiffness is not a constant when the displacement changes. In order to present the change trend of spherical sleeved rubber spring's stiffness objectively, the stiffness of different dimensions of outer radius is calculated under the condition that inner radius is $70 \mathrm{~mm}$. In the table.1, $k_{1}$ is the stiffness when the displacement is $1 \mathrm{~mm}$, and $k_{2}$ is the stiffness when the displacement is $0.1 \mathrm{~mm}$.

Table 1 The change of stiffness

\begin{tabular}{|c|c|c|c|c|c|c|}
\hline The outer radius[mm] & 95 & 105 & 115 & 125 & 135 & 145 \\
\hline$k_{1}[\mathrm{~N} / \mathrm{m}]$ & 21096000 & 10303700 & 6436310 & 3979530 & 2959510 & 2354090 \\
\hline$k_{2}[\mathrm{~N} / \mathrm{m}]$ & 21168500 & 10909800 & 6592380 & 3981580 & 2959680 & 2354520 \\
\hline
\end{tabular}

From table.1 it can be know that the stiffness decreases as the outer radius increases, but the trend of change gradually decreases. Under the different displacements, the stiffness presents a nonlinear 
state. The greater the displacement is, the smaller the stiffness is. And the nonlinear decreases in the case of a large outer radius.

\section{Measurement Results}

Fig.5 is the material object of vector hydrophone. The spherical vector hydrophone is measured by comparing with standard hydrophone in water. Fig.6 are the measurement results. From $315 \mathrm{~Hz}$ to $800 \mathrm{~Hz}$, the receiving sensitivity is about $-205 \mathrm{~dB}$. It accords with the sensitivity characteristics of acoustic vector hydrophone based on geophone. The ration of the maximum and the minimum are more than $20 \mathrm{~dB}$ in the directivity patterns.

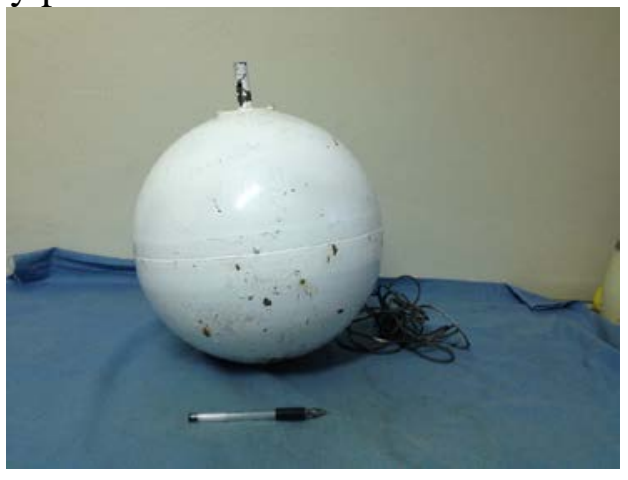

Fig.5 Material object of vector hydrophone
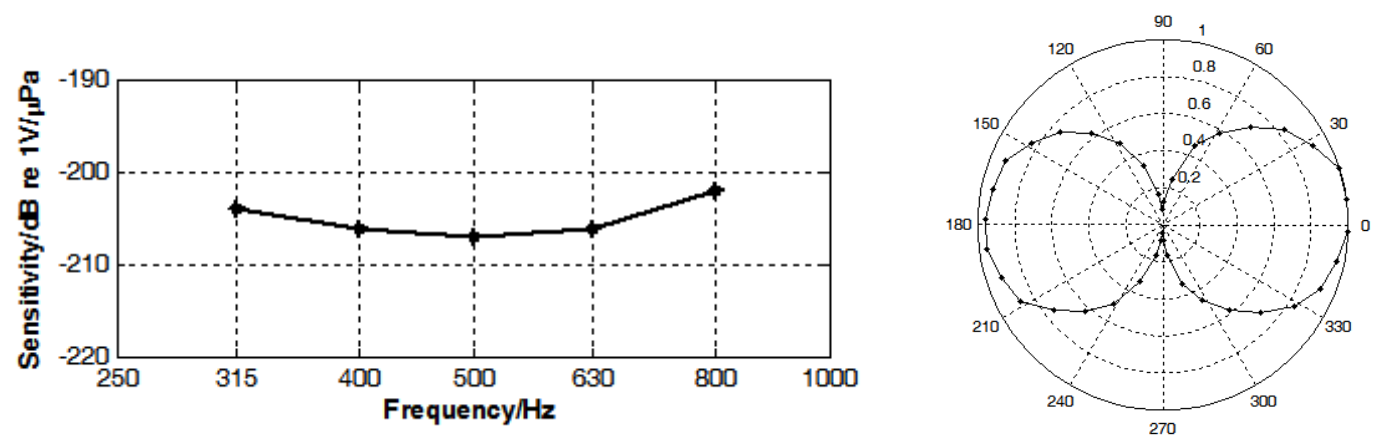

Fig.6 Sensitivity and normalized directivity pattern

\section{Summary}

In this paper, a low frequency spherical acoustic vector hydrophone has been developed by calculating the modal of steel spherical shell and the stiffness of spherical sleeved rubber spring. From the measurement results, this design method is feasible. This kind of acoustic vector hydrophone can sense the low frequency acoustic signals.

\section{References}

[1] M. J. Berliner and J. F. Lindberg: Acoustic Particle Velocity Sensors: Design, Performance, and Applications, edit by American Institute of Physics, Woodbury, NY (1995), in press

[2] Leslie.C.B, Kendall.J.M, Jones.J.L: Jounal of the Acoustical Society of America. Vol.28 (1956), p. $711-715$

[3] James A McConnell: Jounal of the Acoustical Society of America. Vol.113(2003):1395-1400

[4] Zhang Yinghui: Manual of Spring, edit by China Machine Press, BeiJing (2006), in press 